\title{
Transdermal delivery of paeonol using cubic gel and microemulsion gel
}

\author{
This article was published in the following Dove Press journal: \\ International Journal of Nanomedicine \\ 3 August 20II \\ Number of times this article has been viewed
}

\author{
Maofu Luo ${ }^{1,2}$ \\ Qi Shen' \\ Jinjin Chen' \\ 'School of Pharmacy, Shanghai Jiao \\ Tong University, Shanghai, ${ }^{2}$ Jiangxi \\ University of Traditional Chinese \\ Medicine, Nanchang, People's Republic \\ of China
}

Correspondence: Qi Shen

School of Pharmacy, Shanghai Jiao

Tong University, Dongchuan Road 800,

Shanghai 200240, People's Republic of

China

Tel +86 2I 34204049

Fax +86 2I 34204049

Email qshen@sjtu.edu.cn
Background: The aim of this study was to develop new systems for transdermal delivery of paeonol, in particular microemulsion gel and cubic gel formulations.

Methods: Various microemulsion vehicles were prepared using isopropyl myristate as an oil phase, polyoxyethylated castor oil (Cremophor ${ }^{\circledR}$ EL) as a surfactant, and polyethylene glycol 400 as a cosurfactant. In the optimum microemulsion gel formulation, carbomer 940 was selected as the gel matrix, and consisted of $1 \%$ paeonol, $4 \%$ isopropyl myristate, $28 \%$ Cremophor EL/ polyethylene glycol 400 (1:1), and 67\% water. The cubic gel was prepared containing 3\% paeonol, 30\% water, and $67 \%$ glyceryl monooleate.

Results: A skin permeability test using excised rat skins indicated that both the cubic gel and microemulsion gel formulations had higher permeability than did the paeonol solution. An in vivo pharmacokinetic study done in rats showed that the relative bioavailability of the cubic gel and microemulsion gel was enhanced by about 1.51-fold and 1.28-fold, respectively, compared with orally administered paeonol suspension.

Conclusion: Both the cubic gel and microemulsion gel formulations are promising delivery systems to enhance the skin permeability of paeonol, in particular the cubic gel.

Keywords: microemulsion gel, cubic gel, transdermal delivery, paeonol

\section{Introduction}

Paeonol, the active constituent of Cortex moutan, is widely used in traditional Chinese medicine as a pain reliever, anticoagulant, antibiotic, and antiatherosclerotic agent. In recent years, many studies have shown that paeonol has a number of therapeutic effects, including antianalgesic, antihypertensive, anticoagulant, antibacterial, anti-inflammatory, immunoregulatory, antiatherosclerotic, and central nervous system inhibitory activities. ${ }^{1-3}$

A microemulsion is a nanosized formulation in which active drug is dispersed in a mixture of water, oil, and surfactant, frequently in combination with a cosurfactant, and is an optically clear, stable, isotropic liquid, containing particles with diameters of $100 \mathrm{~nm}$ and less. ${ }^{4,5}$ Incorporation of a gel matrix into an o/w microemulsion provides the opportunity to obtain properties comparable with those attainable using hydrogels. Formulations of microemulsion gel were first reported in 1986, ${ }^{6,7}$ and since then their structure and physicochemical properties have been described. ${ }^{8-10}$ Microemulsion gels can increase the permeability of some drugs.

A cubic gel is formed spontaneously when amphiphilic lipids are placed in an aqueous environment. A cubic gel consists of a curved bicontinuous lipid bilayer and has a thermodynamically stable structure. Glyceryl monooleate/water systems 
are an example. They can incorporate small-molecule drugs and large proteins, ${ }^{11,12}$ and have the ability to enhance permeability. ${ }^{13}$

Currently commercialized preparations of paeonol include a cyclodextrin injection, oral solution, and ointment. ${ }^{14}$ Although paeonol preparations of gel and emulsion are being studied, combinations of paeonol with cubic gel and microemulsion gel have been less widely reported. Paeonol is a small phenolic compound showing white crystalline needles. It has the characteristics of a low melting point, volatility, and poor water solubility. The molecular weight of paeonol is 166.18, and its chemical structure is 2-hydroxy-4-methoxy acetophenone (Figure 1). Paeonol is absorbed rapidly after oral administration, and its poor oral bioavailability is suggested to be due to rapid and complete first-pass metabolism of the drug. ${ }^{15}$ Paeonol is more suitable for transdermal delivery. In order to enhance the permeability and stability of paeonol, the microemulsion gel and cubic gel formulations were selected for investigation as drug delivery systems. The purpose of this work was to prepare a microemulsion gel and cubic gel for transdermal delivery of paeonol, to study the skin permeability of paeonol in microemulsion gel and in cubic gel, and evaluate whether the microemulsion gel and cubic gel formulations could improve the bioavailability of paeonol compared with oral administration.

\section{Materials and methods}

\section{Materials}

Paeonol (99\% purity) was obtained from Nanjing Qingze Pharmaceutical Technology Development Co Ltd (Nanjing,

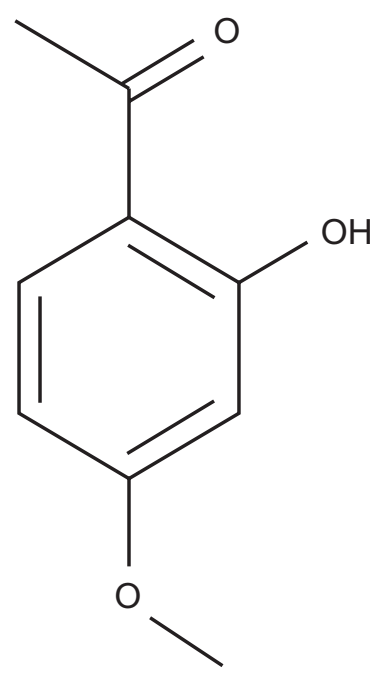

Figure I Structure of paeonol.
China). Glyceryl monooleate ( $>99 \%$ ) was supplied by Danisco (Copenhagen, Denmark). Carbomer 940 was purchased from Shanghai Xietai Chem Co Ltd (Shanghai, China). Transcutol ${ }^{\circledR} \mathrm{P}$ and Labrafac ${ }^{\circledR}$ were kindly donated by Gattefosse (St Priest, France). Cremophor ${ }^{\circledR}$ EL (Crel) and Cremophor ${ }^{\circledR}$ RH 40 (CRH40) were donated by BASF (Ludwigshafen, Germany). Isopropyl myristate, oleic acid, ethyl oleate, propylene glycol, castor oil, ethanol, p-octy polyethylene glycol phenylether, polyethylene glycol 400, and Tween 80 were purchased from Sinopharm Chemical Reagent Corporation (Shanghai, China). Soybean oil was obtained from Tieling North Asia Medicinal Oil Co Ltd (Tieling, China).

\section{Pseudoternary diagrams}

To determine the composition of microemulsions, pseudoternary phase diagrams were constructed by titrating a series of oil, surfactant, and cosurfactant with water mixtures at ambient temperature $\left(25^{\circ} \mathrm{C}\right)$. Surfactants and cosurfactants were blended at different mass ratios (1:2, 1:1, 2:1, 4:1), then each surfactant-cosurfactant mixture was mixed with oil, and the ratio of oil to the surfactant and cosurfactant mixture was varied at 9:1, 8:2, 7:3, 6:4, 5:5, 4:6, 3:7, 2:8, and 1:9(w/w). Water was added drop by drop to the mixture under vigorous stirring. During titration, the isotropic, liquid crystalline, and coarse emulsion phases were observed with turbidityto-transparency or transparency-to-turbidity transitions occurring. Clear and transparent formulations were indicative of a stable microemulsion, and their compositions were recorded. The physical states were marked on a pseudoternary phase diagram representing the surfactant and cosurfactant mixture, with the other two axes representing oil and water.

\section{Preparation of microemulsions}

Based on the phase diagram, different formulae were selected from the microemulsion region (Table 1). Exactly $1 \% \mathrm{w} / \mathrm{w}$ of paeonol was dissolved in the oil, surfactant, and cosurfactant mixtures, and water was added drop by drop under magnetic stirring at room temperature. The control solution was prepared by dissolving paeonol in phosphate-buffered saline at $\mathrm{pH} 7.4$ to achieve a concentration $400 \mu \mathrm{g} / \mathrm{mL}$ and then stirring the solution at $1000 \mathrm{rpm}$.

\section{Microemulsion droplet size and morphology analysis}

Microemulsion particle size was determined using photocorrelation spectroscopy (Malvern, Worcestershire, UK) at 
Table I Compositions of various topical formulations for paeonol delivery

\begin{tabular}{|c|c|c|c|c|c|c|c|c|}
\hline \multirow[t]{2}{*}{ Components } & \multicolumn{8}{|c|}{ Formulations } \\
\hline & A & B & C & D & $\mathbf{E}$ & $\mathbf{F}$ & Cubic gel & MG \\
\hline Ethyl oleate & & & & & & 7 & & \\
\hline Isopropyl myristate & 4 & 4 & 4 & 7 & 10 & & & 4 \\
\hline Crel:PEG400 = I:I & 28 & 39 & 50 & 28 & 28 & 28 & & 28 \\
\hline Water & 67 & 56 & 45 & 64 & 61 & 64 & 30 & 65 \\
\hline Carbomer 940 & & & & & & & & 1 \\
\hline Triethanolamine & & & & & & & & 1 \\
\hline GMO & & & & & & & 67 & \\
\hline Paeonol & I & I & I & I & I & 1 & 3 & 1 \\
\hline
\end{tabular}

Abbreviations: Crel, Cremophor ${ }^{\circledR}$ EL; PEG 400, polyethylene glycol; GMO, glyceryl monooleate; MG, microemulsion gel.

$25^{\circ} \mathrm{C}$. Droplet morphology was observed using transmission electron microscopy (TEM) (JEM-2010; JEOL, Tokyo, Japan). One drop of each diluted sample was deposited on a film-coated 200-mesh copper specimen grid and allowed to stand for 10 minutes, after which any excess fluid was removed with filter paper. The grid was then stained with one drop of $3 \%$ phosphotungstic acid and dried for 5 minutes before examination with an electron microscope.

\section{Preparation of microemulsion gel}

Carbomer 940 0.5\%-2.0\% (w/w) swelled after addition of water, and $\mathrm{pH}$ was adjusted using triethanolamine $0.5 \%-2.0 \%$ $(\mathrm{w} / \mathrm{w})$. The oily solution was prepared by mixing Crel, polyethylene glycol 400, isopropyl myristate, and paeonol, and was then swelled with carbomer 940 and vigorously mixed until a homogeneous dispersion was obtained.

\section{Preparation of cubic gel}

Paeonol 3\% w/w was mixed with melted glyceryl monooleate in glass vials at $42^{\circ} \mathrm{C}$ until completely dissolved or dispersed. Distilled water preheated to $42^{\circ} \mathrm{C}$ was incorporated gradually into the mixture at $30 \% \mathrm{w} / \mathrm{w}$. Samples were kept at room temperature in the dark for 3 days.

\section{In vitro permeability studies}

The abdominal skins of male Wistar rats weighing $220 \pm 20 \mathrm{~g}$ were used for the permeability experiments, which were carried out in accordance with the guidelines of the animal ethics committee at Shanghai Jiao Tong University. The abdominal skin was carefully removed leaving the fat tissue behind. The skin was examined under a magnifying lens for damage or disease, and any skin with a disrupted barrier was excluded. Freshly prepared abdominal skin was used in the diffusion experiments. Vertical Franz-type diffusion cells (Shanghai Institute of Organic Chemistry) with a diffusional surface area of $0.754 \mathrm{~cm}^{2}$ and a $5 \mathrm{~mL}$ cell volume were used to study the permeability of the microemulsion gel and cubic gel formulations. The skin was placed between the donor and receptor compartments of the cells, with the stratum corneum side facing the donor compartment. The receiver compartment was filled with $5 \mathrm{~mL}$ of phosphate-buffered saline at $\mathrm{pH}$ 7.4 , with temperature maintained at $32^{\circ} \mathrm{C} \pm 0.5^{\circ} \mathrm{C}$ using a thermostatic water bath (Variomag, Munich, Germany) and stirring at $600 \mathrm{rpm}$ throughout the experiment. Microemulsion $1 \mathrm{~mL}$ and gel $1 \mathrm{~g}$ samples containing paeonol were placed into each donor compartment, and $0.2 \mathrm{~mL}$ of the receptor solution was withdrawn at predetermined time points (hours 1, 2, 3, $4,5,6,7$, and 8 ), then replaced with an equivalent volume of phosphate-buffered saline to maintained a constant volume. The samples collected were filtered through a $0.45 \mu \mathrm{m}$ membrane and assayed by high-pressure liquid chromatography. The cumulative amount of paeonol that permeated through the rat skins was calculated using the equation:

$$
\begin{gathered}
C_{n c}=C_{n}+(0.2 / 5) \times \Sigma C_{p} \\
Q=C_{n c} \times 5 / 0.754
\end{gathered}
$$

where $Q$ was the accumulated amount at a given time $t, C_{n}$ was the drug concentration of the $n$ sample at the sampling time, $\Sigma C_{p}$ was the sum of the drug concentration of all the n-1 samples measured, and $C_{n c}$ was the transdermal concentration after correction. The cumulative amount $(Q)$ of paeonol permeated was plotted as a function of time $(\mathrm{t})$ for each formulation. The skin permeation rate at steadystate $\left(\mathrm{J}_{\mathrm{ss}}, \mu \mathrm{g} / \mathrm{cm}^{2} /\right.$ hour$)$ was calculated from the slope of the linear portion of the plots of $Q$ versus time. The permeability coefficient was calculated by dividing $\mathrm{J}_{\mathrm{ss}}$ by the initial concentration of drug in the donor cell $\left(\mathrm{C}_{0}\right){ }^{16}$

$$
\text { permeability coefficient }=\mathrm{J}_{\mathrm{ss}} / \mathrm{C}_{0}
$$


The penetration-enhancing effect of paeonol was calculated in terms of the enhancement ratio, and was calculated using the following equation: ${ }^{17}$

$$
\text { enhancement ratio }=\mathrm{K}_{\mathrm{p}} / \mathrm{K}_{0}
$$

where $\mathrm{K}_{\mathrm{p}}$ is the respective formulation and $\mathrm{K}_{0}$ represents the control solution.

\section{In vivo permeability studies}

Male Wistar rats weighing 200-230 g were obtained from the Animal Center of Shanghai Jiao Tong University, and fasted overnight with free access to water before drug administration. Hairs at the abdominal site were shaved off and the skin area washed with distilled water the day before the experiment. Cubic gel and microemulsion gel (30 mg/kg) were applied on the skin surface $\left(7.9 \mathrm{~cm}^{2}\right)$, which was then bound with gauze. Blood samples $(0.25 \mathrm{~mL})$ were collected at hours 0 , $0.5,1.0,2.0,4.0,6.0,8.0,10.0$, and 12.0 after transdermal administration. For the oral pharmacokinetic studies, paeonol was suspended in $0.5 \%$ sodium carboxymethyl cellulose and administered (30 mg/kg) as the control. Blood samples were collected from the jugular vein at $0,0.08,0.25,0.5,0.75,1.0$, 1.5, 2.0, 3.0, 4.0, 6.0, 8.0, and 12.0 hours after dosing.

All collected samples were immediately centrifuged at $4000 \mathrm{rpm}$ for 10 minutes. Plasma $0.1 \mathrm{~mL}$ was added to cinnamic acid $10 \mu \mathrm{L}$ and vortex-mixed with acetonitrile $2 \mathrm{~mL}$ for 1 minute and centrifuged at 10,000 rpm for 10 minutes. The supernatant was transferred to a clean test tube and evaporated to dryness at $25^{\circ} \mathrm{C}$ under a stream of nitrogen. The residue was dissolved in methanol $1 \mathrm{~mL}$, and $20 \mu \mathrm{L}$ of the sample solution was injected into the high-pressure liquid chromatography system.

\section{Analysis of paeonol}

Paeonol was analyzed using a reversed phase high-pressure liquid chromatography system. The chromatographic system consisted of a Waters 717 Plus autosampler (Waters, Milford, MA) and a Waters 2487 dual $\lambda$ absorbance detector equipped with a prepacked Hypersil ODS column $(150 \mathrm{~mm} \times 4.6 \mathrm{~mm}$, $5 \mu \mathrm{m}$, Dalian Elite Analytical Instruments Co Ltd, Dalian, China). The mobile phase for analysis of paeonol consisted of methanol, water, and acetic acid (53:47:0.01, v/v). The eluent was pumped at a flow rate of $1.0 \mathrm{~mL} / \mathrm{min}$. The detector wavelength was set at $274 \mathrm{~nm}$. The injection volume was $20 \mu \mathrm{L}$.

\section{Statistical analysis}

All skin permeability experiments were repeated three times, and the results expressed as means \pm standard deviation.
Statistical significance was assessed using the Student's $t$-test for multiple comparison. A $P$ value $<0.05$ was considered to indicate statistical significance.

\section{Results and discussion Pseudoternary diagrams}

Pseudoternary phase diagrams of the system containing isopropyl myristate, Crel, polyethylene glycol 400, and water are shown in Figure 2. The black areas indicate the clear $\mathrm{o} / \mathrm{w}$ microemulsion region. The microemulsion domain was determined by visual inspection for clarity and fluidity as well as through a cross polarizer for the absence of a liquid crystalline phase. The rest of the region in the phase diagram represents turbid and conventional emulsions based on visual observation. No liquid crystalline structure was observed using the cross polarizer. Phase studies were used to estimate the effect of surfactant/cosurfactant $(\mathrm{S} / \mathrm{Cos})$ in stable o/w microemulsion regions. As shown in Figure 2, the monophasic zone of $\mathrm{o} / \mathrm{w}$ microemulsion reaches a maximum at a $\mathrm{S} / \mathrm{Cos}$ of 1 .

\section{Characteristics and physicochemical properties of the microemulsions}

The particle size of the microemulsion was one of the most important properties. All the microemulsions (A-F) had small average droplet diameters of $27-60 \mathrm{~nm}$. As a result, the droplet size of formulation A was $39.80 \pm 4.6 \mathrm{~nm}$. The morphology of microemulsion formulation A was characterized using transmission electron microscopy (Figure 3), and showed a spherical shape and uniform droplet size. Samples were diluted with distilled water before testing to avoid multiscattering phenomena. After that, the droplet size of the diluted microemulsion was not significantly changed. The mean particle size remained consistent for 3 months at room temperature.

\section{Preparation of gels}

In this work, carbomer 940 was added to vehicle A to prepare the microemulsion gel. We found that carbomer 940 could increase the viscosity of the microemulsion, maintain the microemulsion structure, and provide a good matrix for the microemulsion gel.

Microemulsion gel containing $0.5 \%$ carbomer 940 had relatively high fluidity. The viscosity of the microemulsion gel increased with increasing concentrations of carbomer 940. However, $2 \%$ carbomer 940 resulted in excessive microemulsion gel viscosity. Microemulsion gel containing 1\% carbomer 940 has suitable viscosity for topical application. ${ }^{18}$ 


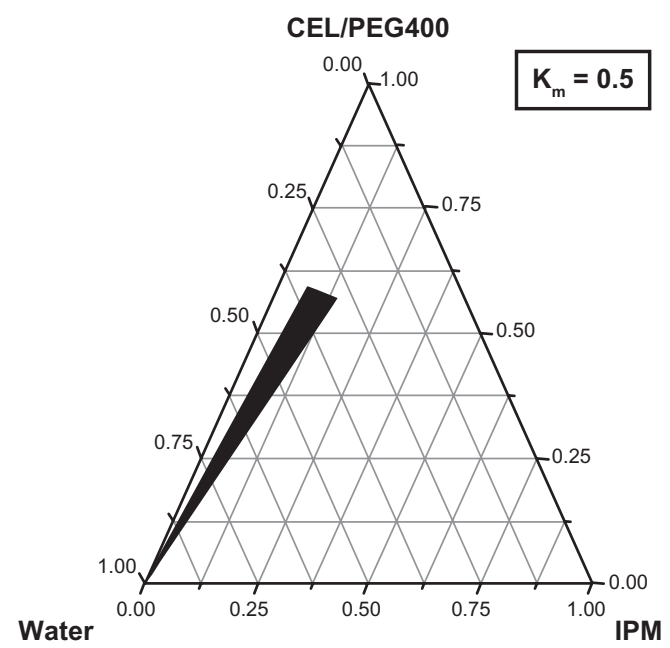

CEL/PEG400

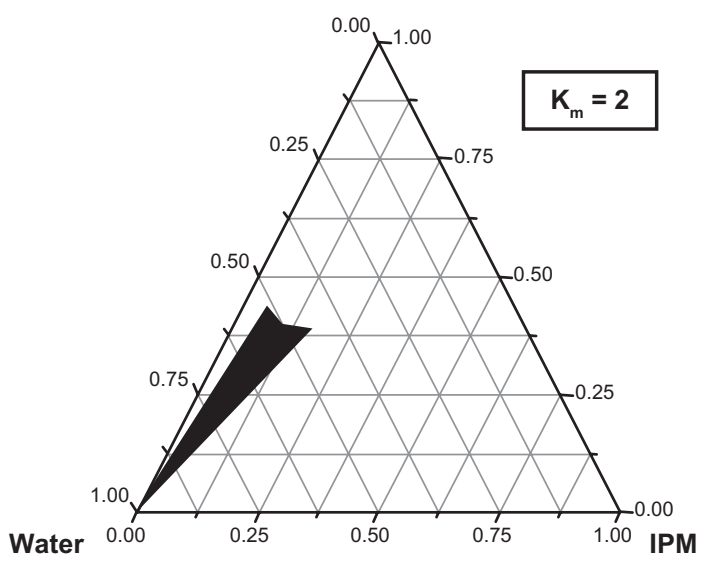

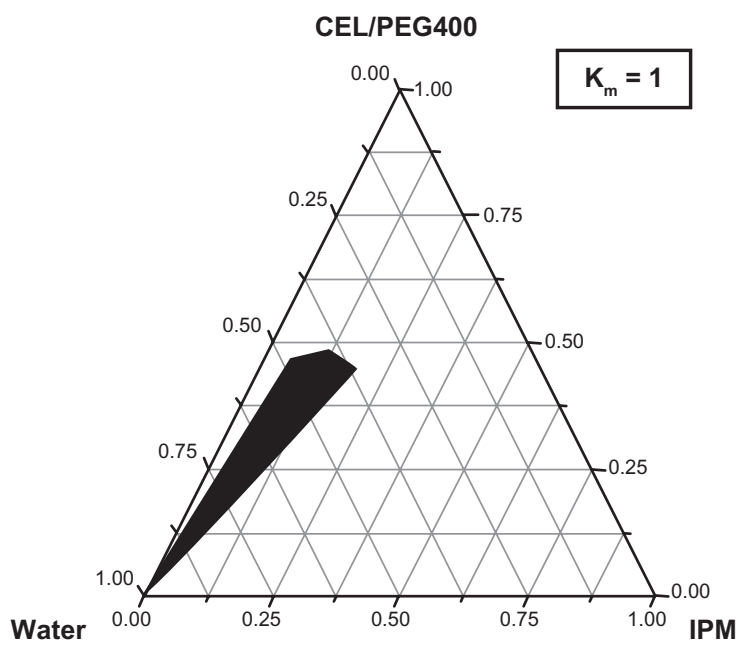

CEL/PEG400

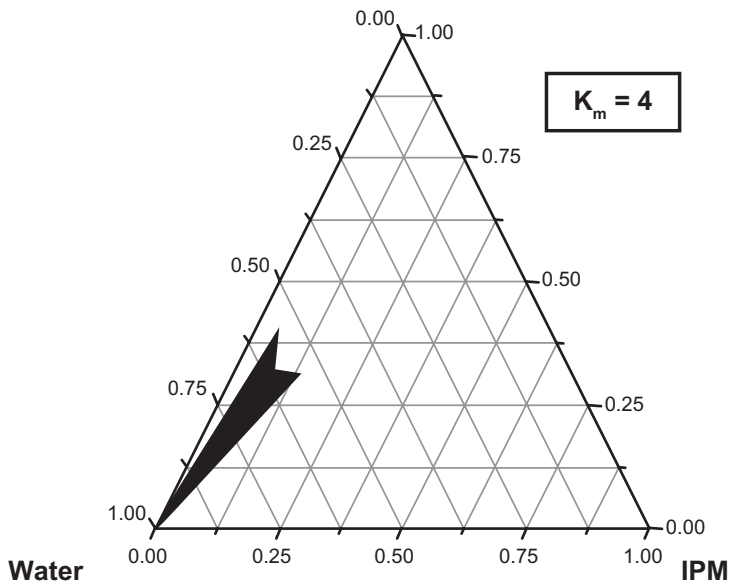

Figure 2 Pseudoternary phase diagrams of microemulsion composed of isopropyl myristate, surfactant (Crel), cosurfactant (PEG400) and water. Note: Black area represents o/w microemulsion region; $\mathrm{K}_{\mathrm{m}}=\mathrm{S} / \mathrm{Cos}$.

Abbreviations: CEL, Cremophor EL ${ }^{\circledR}$, polyoxyethylated castor oil; Crel, Cremophor EL; IPM, isopropyl myristate; o/w, oil in water; PEG 400, polyethylene glycol.

As far as we know, glyceryl monooleate, monoolein, phosphatidylethanolamine, phospholipids, and PEGylated phospholipids ${ }^{19-22}$ are used to form the cubic phases. In this study, glyceryl monooleate was selected to prepare the cubic gel. After 3 days of equilibration, the cubic gel developed into glass-clear, nonbirefingent, and stiff cubic phase forms, and had greater transparency than the microemulsion gel. Both microemulsion gel with $1 \%$ carbomer 940 and the cubic gel were stable at $30^{\circ} \mathrm{C}$. No changes in phase separation or paeonol degradation were observed over 3 months. The centrifuge test showed that all the gels still maintained good physical stability.

\section{In vitro permeability studies}

In vitro skin studies were performed to compare drug permeability from six different microemulsion formulations, and the percutaneous parameters, including permeability coefficient and enhancement ratio, were calculated following zero order release kinetics. A steady increase in the amount of permeated drug in the receptor compartment with time was observed. The permeation coefficient ranged from $1.68 \pm 0.31$ to $6.53 \pm 1.08 \mathrm{~cm} /$ hour $\times 10^{-3}$, and the highest value was for vehicle $A$ and the lowest was for vehicle $C$, so vehicle A was selected to prepare the microemulsion gel.

As shown in Table 2, the permeability coefficient of the microemulsion gel was $5.88 \pm 0.28 \mathrm{~cm} /$ hour $\times 10^{-3}$, which was less than that for vehicle $\mathrm{A}$. Vehicle $\mathrm{A}$ and the microemulsion gel produced a significantly higher permeability coefficient, ie, a 2.13-fold and 1.92-fold increase, respectively, compared with the control solution. Peltola et al reported that carbomer 940 might influence the permeability of estradiol. The permeability of paeonol decreased with the addition of carbomer 


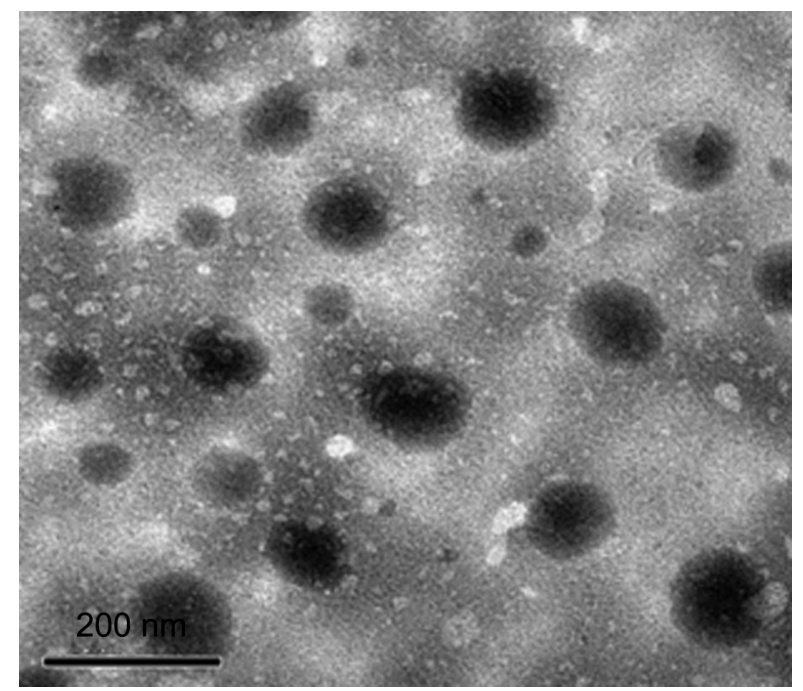

Figure 3 Transmission electron microphotography of paeonol microemulsion.

940 to the microemulsion, possibly because of increased viscosity and the changing structure of the vehicle. ${ }^{23,24} \mathrm{In}$ this work, the addition of $1 \%$ carbomer 940 had the same influence on the permeability of paeonol.

Figure 4 shows the permeability of paeonol through rat skin. The cumulative amount of paeonol in the cubic gel was significantly higher $(P<0.05)$ than that of paeonol in the microemulsion gel and control solution. With a permeability coefficient of $8.34 \pm 0.49 \mathrm{~cm} /$ hour $\times 10^{-3}$, cubic gel was a better carrier for transdermal delivery of paeonol than the microemulsion gel and control solution. There may be two factors accounting for this. Firstly, the content of paeonol in the cubic gel was higher than in the microemulsion gel, which means that the cubic gel had better drug solubility, which could contribute to improved absorption of the drug..$^{25,26}$ Secondly, the structure of the cubic gel might be better than that of the microemulsion gel for drug delivery. The structure of the cubic phase is unique and consists of a curved bicontinuous lipid bilayer, which resembles the structure of lipid membranes found in nature, ${ }^{27,28}$ so might result in good adhesiveness to the skin and good delivery of the drug.

Table 2 Permeability coefficient and enhancement ratio of paeonol through excised rat skin from different vehicles

\begin{tabular}{lll}
\hline Vehicle & $\mathbf{K}_{\mathbf{p}}(\mathbf{c m} /$ hour $) \times 1 \mathbf{0}^{-3}$ & Enhancement ratio \\
\hline Control & $3.06 \pm 0.10$ & \\
A & $6.53 \pm 1.09^{*}$ & 2.13 \\
MG & $5.88 \pm 0.28^{*}$ & 1.92 \\
Cubic gel & $8.34 \pm 0.49^{* *}$ & 2.73 \\
\hline
\end{tabular}

Notes: ${ }^{*} P<0.05$, $* * P<0.01$, compared with control; each value represents means \pm standard deviation of at least three experiments.

Abbreviation: MG, microemulsion gel.

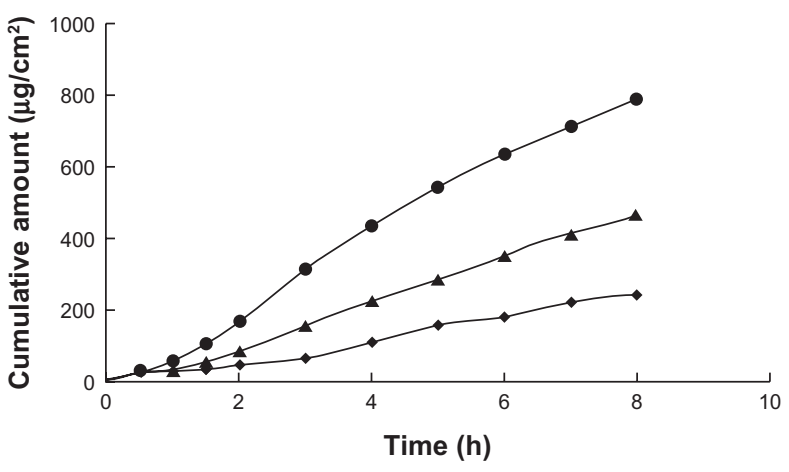

Figure 4 The cumulative amount of paeonol from cubic gel (-๑-) and microemulsion gel (- $\mathbf{\Delta}-)$ and control solution (- - -).

Note: Results are expressed as means \pm standard deviation of at least three experiments.

\section{In vivo permeability studies}

The mean plasma concentration versus time profiles for paeonol after oral and transdermal administration are shown in Figure 5, and the mean pharmacokinetic parameters calculated by DAS2.1.1 are given in Table 3. These show that paeonol is rapidly absorbed, with a maximum concentration of $1.64 \pm 0.14 \mu \mathrm{g} / \mathrm{mL}$ at $0.25 \pm 0.06$ hours after a single oral dose. The time taken to reach maximum concentration of paeonol in the cubic gel and microemulsion gel was 3 hours and 2 hours, respectively, after transdermal administration. This result is similar to the phenomenon reported by Sun et al, who detected the maximum concentration at 5 minutes after an oral dose. ${ }^{29}$ This was due to dermal accumulation of paeonol because of the barrier properties of skin. An increase in the area under the concentration-time curve (AUC) for paeonol was observed when the cubic gel and microemulsion gel were studied. The relative bioavailability of paeonol in cubic gel and microemulsion gel was 1.51 and 1.28 times higher, respectively, than after oral administration of the drug. Mean residence times following a transdermal dose of cubic gel and microemulsion gel were $5.63 \pm 1.32$ hours and $5.62 \pm 1.11$ hours, respectively, which were higher than that for paeonol suspension.

Compared with oral administration, transdermal administration had a longer time to reach maximum plasma concentration, a lower maximum plasma concentration, higher AUC, and higher mean residence time, because of avoidance of the substantial amount of hepatic first-pass metabolism associated with oral administration.

The mechanism for the enhanced transdermal penetration of microemulsion gel and cubic gel for paeonol might be attributable to several factors. Firstly, the high concentration (1\%) of paeonol in the microemulsions resulted in high concentration gradients, which might be the main mechanism 


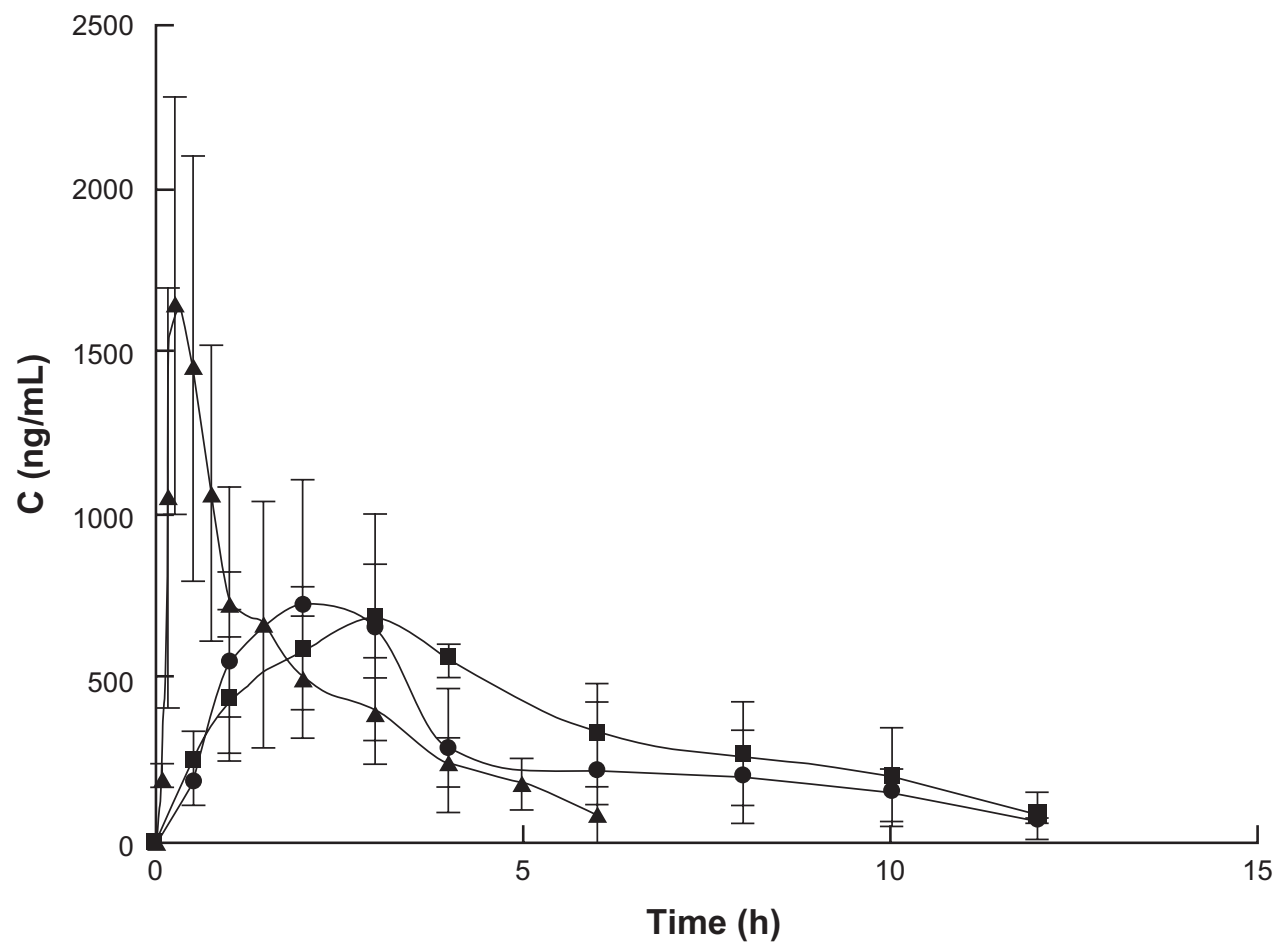

Figure 5 Plasma concentration of paeonol following oral and transdermal administration to rats.

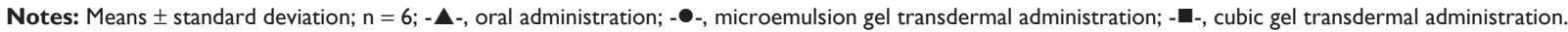

by which paeonol in a microemulsion gel penetrates the skin. ${ }^{26}$

Microemulsions could act as drug reservoirs whereby drug is released from an inner phase to an outer phase and then into the skin. ${ }^{24}$ Secondly, due to the small droplet size, droplets settling in close contact with the skin might penetrate the skin surface. ${ }^{24}$

In the case of cubic phase gel, glyceryl monooleate could intercalate with the extracellular lipid matrix of the skin, and disrupt its well organized compact structure. ${ }^{30}$ Some studies have reported that enhanced transdermal delivery of drugs may depend on a disordered state, or depend on a different

Table 3 Pharmacokinetic parameters obtained after oral and transdermal administration of paeonol in rats

\begin{tabular}{|c|c|c|c|}
\hline \multirow[t]{2}{*}{ Parameter } & \multicolumn{2}{|c|}{ Transdermal administration } & \multirow{2}{*}{$\begin{array}{l}\text { Oral } \\
\text { administration }\end{array}$} \\
\hline & Cubic gel & MG & \\
\hline$C_{\max }(\mu g / m L)$ & $0.68 \pm 0.10 *$ & $0.72 \pm 0.15^{*}$ & $1.64 \pm 0.14$ \\
\hline $\mathrm{T}_{\max }$ (hours) & $3.00 \pm 0.16^{* *}$ & $2.00 \pm 0.32^{* *}$ & $0.25 \pm 0.06$ \\
\hline $\mathrm{T}_{1 / 2}$ (hours) & $2.58 \pm 0.13$ & $3.45 \pm 0.49 * *$ & $1.54 \pm 0.29$ \\
\hline 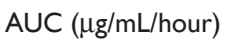 & $4.60 \pm 0.54 *$ & $3.89 \pm 1.01$ (ns) & $3.04 \pm 0.42$ \\
\hline MRT (hours) & $5.63 \pm 1.32 *$ & $5.62 \pm 1.11 *$ & $1.64 \pm 0.33$ \\
\hline
\end{tabular}

Notes: Data are expressed as the means \pm standard deviation for six rats; $* P<0.05$, compared with oral administration; $P<0.01$, compared with oral administration. Abbreviations: $A \cup C$, area under the concentration-time curve; $M G$, microemulsion gel; $C_{\max }$, maximum concentration; $n s$, not significant; $T_{\max }$, time to reach maximum concentration; $T_{1 / 2}$, elimination half-life; MRT, mean residence time. order of lipids, given that intercellular lipids can rearrange into a cubic phase under specific circumstances. ${ }^{31}$

In both the in vitro permeability and in vivo bioavailability experiments, a correlation was obtained between the outcomes of the in vitro permeability model and the in vivo bioavailability data. The in vitro permeability experiments indicate that the cubic gel formulation yielded the highest permeability coefficient of the three tested formulations, and this was also the case in the in vivo assessment model, with the AUC of the cubic gel formulation being higher than that of the other two formulations.

\section{Conclusion}

In this study, novel microemulsion gel and cubic gel formulations for transdermal delivery of paeonol were developed. Our results indicate that both the cubic gel and microemulsion gel formulations had a higher permeability coefficient than the control group. An in vivo pharmacokinetic study in rats showed that the relative bioavailability of the cubic gel and the microemulsion gel was enhanced by about 1.51-fold and 1.28-fold, respectively, compared with oral administration of paeonol suspension. These data collectively support the hypothesis that cubic gel and microemulsion gel formulations are promising delivery systems to enhance the skin permeability of paeonol, especially the cubic gel. 


\section{Acknowledgments}

The work was supported by the Shanghai Municipal Committee of Science and Technology (grant No. 08DZ1971304), the National Basic Research Program of China (No. 2007CB936004), and the National Pharmaceutics Technology Platforms for Innovative Drugs (No. 2009ZX09310-007).

\section{Disclosure}

The authors report no conflicts of interest in this work.

\section{References}

1. Riley CM, Ren TC. Simple method for the determination of paeonol in human and rabbit plasma by high-performance liquid chromatography using solid-phase extraction and ultraviolet detection. J Chromatogr. 1989;489:432-437.

2. Chou TC. Anti-inflammatory and analgesic effects of paeonol in carrageenan-evoked thermal hyperalgesia. Br J Pharmacol. 2003;139: $1146-1152$.

3. Kim SH, Kim SA, Park MK, Kim SH, Park YD, Na HJ. Paeonol inhibits anaphylactic reaction by regulating histamine and TNF-alpha. Int Immunopharmacol. 2004;4:279-287.

4. El Maghraby GM. Transdermal delivery of hydrocortisone from eucalyptus oil microemulsion: effects of cosurfactants. Int $J$ Pharm. 2008;355:285-292.

5. Kawakami K, Yoshikawa T, Hayashi T, Nishihara Y, Masuda K. Microemulsion formulation for enhanced absorption of poorly soluble drugs: II. In vivo study. J Control Release. 2002;81:75-82.

6. Haering G, Luisi PL. Hydrocarbon gels from water-in-oil microemulsions. J Phys Chem. 1986;90:5892-5895.

7. Quellet C, Eicke HF. Mutual gelation of gelatin and water-in oil microemulsions. Chimia (Aarau).1986;40:233-238. German.

8. Atkinson PJ, Robinson BH, Howe AM, Heenan RK. Structure and stability of microemulsion-based organo-gels. J Chem Soc Faraday Trans. 1991;87:3389-3397.

9. Rees GD, Nascimento MG, Jenta TR, Robinson BH. Reverse enzyme synthesis in microemulsion-based organo-gels. Biochim Biophys Acta. 1991;1073:493-501.

10. Jenta TR, Batts G, Rees GD, Robinson BH. Biocatalysis using gelatin microemulsion-based organogels containing immobilized Chromobacterium viscosum lipase. Biotechnol Bioeng.1997;53: 121-131.

11. Norling T, Landing P, Larsson K, Krog N, Nissen SS. Formulation of a drug delivery system based on a mixture of monoglycerides and triglycerides for use in the treatment of periodontal disease. $J$ Clin Periodontol. 1992;19:687-692.

12. Geraghty PB, Attwood D, Collet JH, Dandiker Y. The in vitro release of some antimuscarinic drugs from monoolein/water lyotropic liquid crystalline gels. Pharm Res.1996;13:1265-1271.

13. Han IH, Choi SU, Nam DY, et al. Identification and assessment of permeability enhancing vehicles for transdermal delivery of glucosamine hydrochloride. Arch Pharm Res. 2010;33:293-299.
14. Xi J, Liu S, Liu C. The study of paeonol formulation and clinical application. Lishizhen Medicine and Materia Medica Research. 2005; 16:66.

15. Wu X, Chen H, Chen X, Hu Z. Determination of paeonol in rat plasma by high-performance liquid chromatography and its application to pharmacokinetic studies following oral administration of Moutan cortex decoction. Biomed Chromatogr. 2003;17:504-508.

16. Modamio P, Lastra CF, Mariño EL. Transdermal absorption of celiprolol and bisoprolol in human skin in vitro. Int J Pharm. 1998;173: 141-148.

17. Babar A, Solanki UD, Cutie AJ. Piroxicam release from dermatological bases: In vitro studies using cellulose membrane and hairless mouse skin. Drug Dev Ind Pharm. 1990;16:523-540.

18. Lapasin R, Grassi M, Coceani N. Effects of polymer addition on the rheology of o/w microemulsions. Rheol Acta. 2001;40:185-192.

19. Leslie SB, Puvvada S, Ratna BR, Rudolph AS. Encapsulation of hemoglobin in a bicontinuous cubic phase lipid. Biochim Biophys Acta. 1996;1285:246-254.

20. Tenchov B, Rappolt M, Koynova R, Rapp G. New phases induced by sucrose in saturated phosphatidylethanolamines: an expanded lamellar gel phase and a cubic phase. Biochim Biophys Acta. 1996;1285: 109-122.

21. Eriksson PO, Lindblom G. Lipid and water diffusion in bicontinuous cubic phases measured by NMR. Biophys J. 1993;64:129-136.

22. Koynova R, Tenchov B, Rapp G. Low amounts of PEG-lipid induce cubic phase in phosphatidylethanolamine dispersions. Biochim Biophys Acta. 1997;1326:167-170.

23. Trotta M. Influence of phase transformation on indomethacin release from microemulsions. J Control Release. 1999;60:399-405.

24. Peltola S, Saarinen-Savolainen P, Kiesvaara J, Suhonen TM. Microemulsions for topical delivery of estradiol. Int J Pharm. 2003;254: 99-107.

25. Park ES, Chang SY, Hahn M, Chi SC. Enhancing effect of polyoxyethylene alkyl ethers on the skin permeation of ibuprofen. Int J Pharm. 2000;209:109-119.

26. Chen HB, Chang XL, Du DR, Xu HB, Yang XL. Microemulsion-based hydrogel formulation of ibuprofen for topical delivery. Int $J$ Pharm. 2006;315:52-58.

27. Larsson K, Fontell K, Krog N. Structural relationships between lamellar, cubic and hexagonal phases in monoglyceride-water systems. Possibility of cubic structures in biological systems. Chem Phys Lipids. 1980;27: 321-328.

28. Larsson K. Cubic lipid-water phases: Structures and biomembrane aspects. J Phys Chem. 1989;93:7301-7314.

29. Sun YC, Sun GP, Shen YX, Chen LM, Qu J, Wei W. Study the pharmacokinetics of paeonol in mice in vivo. Chin Hosp Pharm J. 2006;26: 543-545.

30. Kwon TK, Kim JC. In vitro skin permeation of monoolein nanoparticles containing hydroxypropyl beta-cyclodextrin/minoxidil complex. Int $J$ Pharm. 2010;392:268-273.

31. Foldvari M, Badea I, Wettig S, et al. Topical delivery of interferon alpha by biphasic vesicles: Evidence for a novel nanopathway across the stratum corneum. Mol Pharm. 2010;7:751-762.
International Journal of Nanomedicine

\section{Publish your work in this journal}

The International Journal of Nanomedicine is an international, peerreviewed journal focusing on the application of nanotechnology in diagnostics, therapeutics, and drug delivery systems throughout the biomedical field. This journal is indexed on PubMed Central, MedLine, CAS, SciSearch ${ }^{\circledR}$, Current Contents ${ }^{\circledR} /$ Clinical Medicine,

\section{Dovepress}

Journal Citation Reports/Science Edition, EMBase, Scopus and the Elsevier Bibliographic databases. The manuscript management system is completely online and includes a very quick and fair peer-review system, which is all easy to use. Visit http://www.dovepress.com/ testimonials.php to read real quotes from published authors. 